EPJ Web of Conferences 95,02006 (2015)

DOI: $10.1051 /$ epjconf/ 20159502006

(C) Owned by the authors, published by EDP Sciences, 2015

\title{
High-density matter: current status and future challenges
}

\author{
J. R. Stone $e^{1,2, a}$ \\ ${ }^{1}$ Department of Physics, University of Oxford, Parks Road, OX1 3PU Oxford, UK \\ ${ }^{2}$ Department of Physics and Astronomy, University of Tennessee, Knoxville, TN 37996-1200, USA
}

\begin{abstract}
There are many fascinating processes in the Universe which we observe in more and more in detail thanks to increasingly sophisticated technology. One of the most interesting phenomena is the life cycle of stars, their birth, evolution and death. If the stars are massive enough, they end their lives in the core-collapse supernova explosion, the one of the most violent events in the Universe. As the result, the densest objects in the Universe, neutron stars and/or black holes are created. Naturally, the physical basis of these events should be understood in line with observation. The current status of our knowledge of processes in the life of stars is far from adequate for their true understanding. We show that although many models have been constructed their detailed ability to describe observations is limited or non-existent. Furthermore the general failure of all models means that we cannot tell which are heading in the right direction. A possible way forward in modeling of high-density matter is outlined, exemplified by the quark-meson-coupling model (QMC). This model has a natural explanation for the saturation of nuclear forces and depends on very few adjustable parameters, strongly constrained by the underlying physics. Latest QMC results for compact objects and finite nuclei are presented.
\end{abstract}

\section{Introduction}

The structure of matter at high density and temperature is one of the central questions of current theoretical physics. Answering this question requires a joint effort of nuclear, particle and astrophysics theorists, combined with tests based on the most advanced astrophysical observation data and relevant terrestrial experiments.

In the generally accepted QCD phase diagram, expressed in terms of temperature $\mathrm{T}$ and baryon number density (Figure 1), two extreme regions can be identified. At low (zero) temperature and high baryon number density (several times higher that the saturation density of symmetric nuclear matter) the best representation of nuclear matter can be found in the cores of cold neutron stars and, at somewhat higher temperature, in core-collapse supernovae. The other extreme is at low (zero) baryon number density and extremely high temperature (several hundred $\mathrm{MeV}$ ). This region of the phase diagram corresponds to conditions in the early universe. The adjacent region with lower temperatures and somewhat higher baryonic densities can, in principle, be probed in existing terrestrial heavy ion collisions, at LHC, RHIC and GSI. Extension towards even higher chemical potentials and lower temperatures is expected in planned facilities FAIR at GSI and NICA in Dubna. One of the key questions is the location of the critical point where the hadronic and quark-gluon phases coexist. There is an extensive beam scan campaign at RHIC, performed by the STAR collaboration, in this direction, but no final conclusion has been reached as yet. The position of the critical point in the QCD phase diagram has important implications for the location of the hadronic-quark phase transition in neutron stars and core-collapse supernovae.

a e-mail: j.stone@physics.ox.ac.uk 


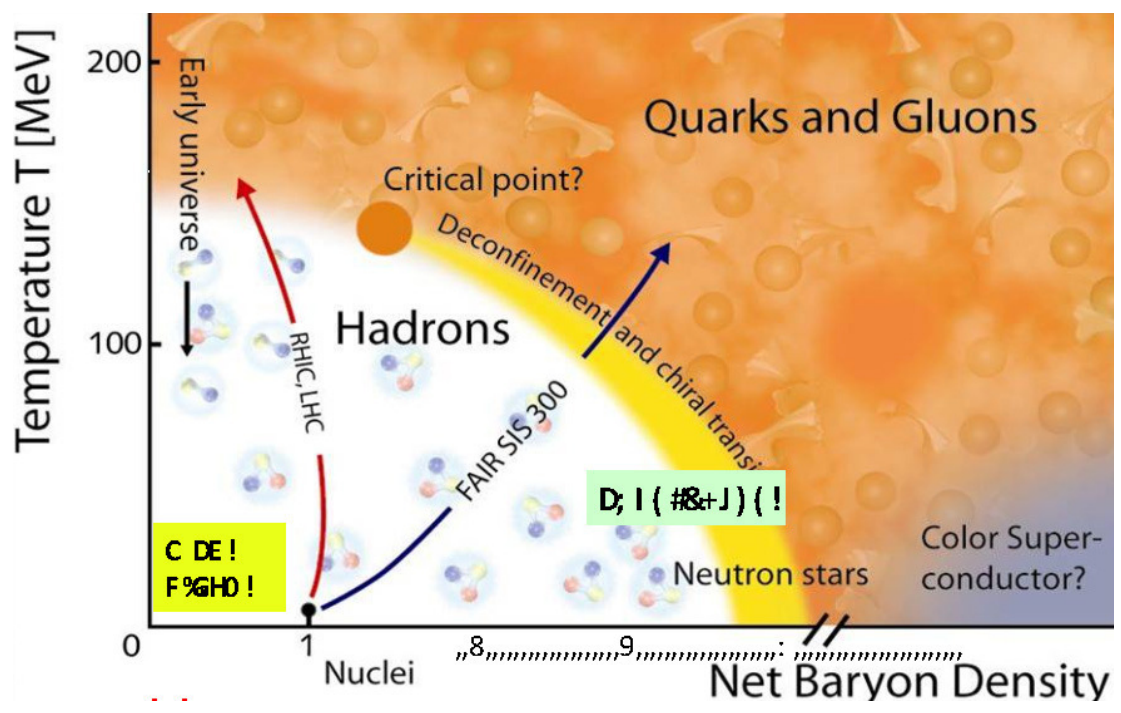

Figure 1. QMC phase diagram adopted from www.gsi.de showing temperature and baryon density range for different phases of hadronic and quark matter.

In this paper we address the fundamental question as to whether we have enough experimental and observational data to compare with a current theory to give answers about questions concerning properties of high-density matter in the low temperature and high baryonic density region of the QMC diagram. In Section 2 we introduce the definiton of the Equation of State (EoS) and its connection with nuclear and particle models. Section 3 is devoted to the up-to-date constraints on the EoS from observation of neutron stars and core-collapse supernovae. Constraints coming from terrestrial experiments with heavy-ion collisitons and hypernuclei, as well as, potentially, from lattice QCD, are briefly discussed in Section 4, followed by discussion of the Quark-Meson-Coupling (QMC) model, which offers a new perspective in description of low energy nuclear and particle phenomena in Section 5. Summary and conclusions are presented in Section 6.

\section{Equation of state of high-density matter}

The key property of high-density matter is the Equation of State (EoS). Starting from the Boltzmann theory of ideal gases, the relation between the pressure $\mathrm{P}$, energy density $\varepsilon$ and temperature $\mathrm{T}$ of matter, can be derived. We have

$$
P=\rho^{2}\left(\frac{\partial(\varepsilon / \rho)}{\partial \rho}\right)_{s / \rho} \quad \varepsilon(\rho, T)=\sum_{f} \varepsilon_{f}(\rho, T)
$$

where $\rho$ is particle number density, $\varepsilon$ is the energy per particle and the summation carries over all $f$ constituents present in the matter. The two key points here are: (i) the EoS critically depends on constituents of the matter and the interactions between them and (ii) $\varepsilon$ is unknown and can only be determined from nuclear and/or particle physics models. Limitations of these models are the main source of ambiguity in theoretical determination of the EoS of high-density matter. There are many variants of microscopic and phenomenological models of hadronic matter with different levels of complexity. To name a few, mean-field non-relativistic and relativistic models, ab initio models with two- and three-body nucleon-nucleon interactions and Quark-Meson Coupling models are frequently used [1-5]. There is also a wide choice of composition of hadronic matter, from nucleons only to 
matter including the full baryon octet and baryon resonances (p, $\mathrm{n}, \Lambda, \Sigma, \Xi, \Delta)$, and mesons $(\pi, \mathrm{K}, \mathrm{H}-$ dibaryon condensates). Deconfined ( $\mathrm{u}, \mathrm{d}, \mathrm{s})$ matter has been proposed, which ought to be in a color superconducting state [1,2]. Models of quark matter are even less reliable and range from the different forms of the MIT bag, Nambu-Jona-Lasinio, Chromo-Dielectric, Dyson-Schwinger, perturbative approach to QCD and implementation of the Polyakov-loop technique at non-zero temperature. There are many models of phases of color-superconductivity in quark matter. We illustrate possible components of high-density matter in Figure 2.

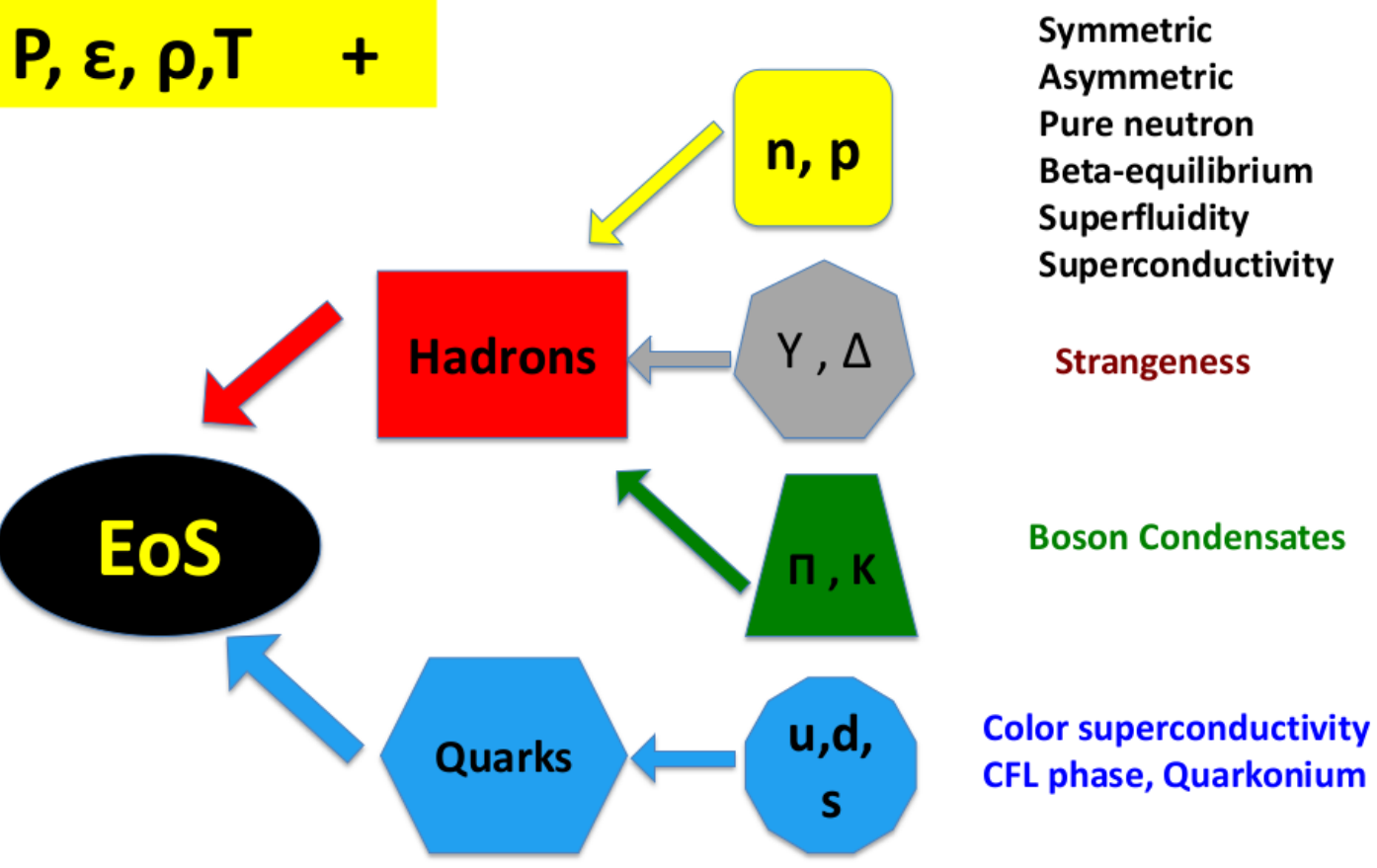

\section{Hybrids, phase transitions, threshold conditions}

Figure 2. A cartoon demonstrating main theoretically possible constituents present in the interior of neutron stars and cores of collapsing massive stars. It is important to realize that in a full theoretical description of these objects also threshold conditions, phase transitions and hybrid configurations must be taken into account.

If all the multifaceted physics of high-density matter were known, it would determine a "universal" EoS including all the components and their interactions allowed by physical laws. However, such EoS can never manifest itself fully. Our observations are only sensitive to projections of the EoS to particular sectors (degrees of freedom), which can be limited by observation and/or measurement. The long- term aim of our research is clearly to describe all these sectors on the same physical basis, which would allow full control of the models and ensure the most detailed description and best predictive power possible.

The current situation is however still far from this ideal situation. The lack on knowledge of forces acting amongst the elementary particles leads to models with a large number of parameters, too many to be determined unambiguously from experiments and observation. For example, Dutra et al. [6] collected 240 non-relativistic mean-field models based on the Skyrme interaction, the effective density dependent force, determined by the aid of 13-15 adjustable parameters, and assessed their ability to satisfy a series of criteria derived from macroscopic properties of nuclear matter in the 
vicinity of nuclear saturation density at zero temperature and their density dependence, derived by the liquid-drop model, in experiments with giant resonances and heavy-ion collisions. Out of the 240 models, only sixteen were shown to satisfy all these constraints. Additional, more microscopic, constraints on the density dependence of the neutron and proton effective mass in $\beta$-equilibrium matter, Landau parameters of symmetric and pure neutron matter, and observational data on high- and low-mass cold neutron stars further reduced this number to five, a very small group of recommended Skyrme parameterizations to be used in future applications of the Skyrme interaction of nuclearmatter-related observables. Disappointingly, the five successful parameter sets show no common features being arrived at by fitting different observational and experimental properties and it is impossible to judge which of is to be preferred.

Very recently, a set of 263 relativistic mean-field (RMF) models was examined in a similar fashion [7]. Three different sets of constraints related to symmetric nuclear matter, pure neutron matter, symmetry energy, and its derivatives were used. The first set (SET1) was the same as used in assessing the Skyrme parameterizations was not satisfied by any RMF models. The second and third sets (SET2a and SET2b) were more suitable for analysis of RMF and included up-to-date theoretical experimental and empirical information. The sets of updated constraints (SET2a and SET2b) differed somewhat in the level of restriction but still yielded only 4 and 3 approved RMF models, respectively. The results changed dramatically if the constraint on the volume part of the isospin incompressibility $\left(\mathrm{K}_{\tau, \mathrm{V}}\right)$ was eliminated, when there were 35 approved models in SET2a and 30 in SET2b. However, there is no physical reason to eliminate this constraint and it should rather be used to identify and eliminate an apparent systematic deficiency in RMF models of nuclear matter. Again, the parameters of the successful models are largely empirical so that definitive information is gained as to the underlying physics.

Mean field models discussed above are usually constructed with the constraint that basic properties of symmetric nuclear matter (SNM) (equal number of protons and neutrons, no Coulomb interaction), namely saturation energy $\mathrm{E}_{0} \sim-16 \mathrm{MeV}$ at density $\rho_{0}=0.16 \mathrm{fm}^{-3}$ be predicted correctly. This requirement is not imposed on another class of models, which start from a bare two- body
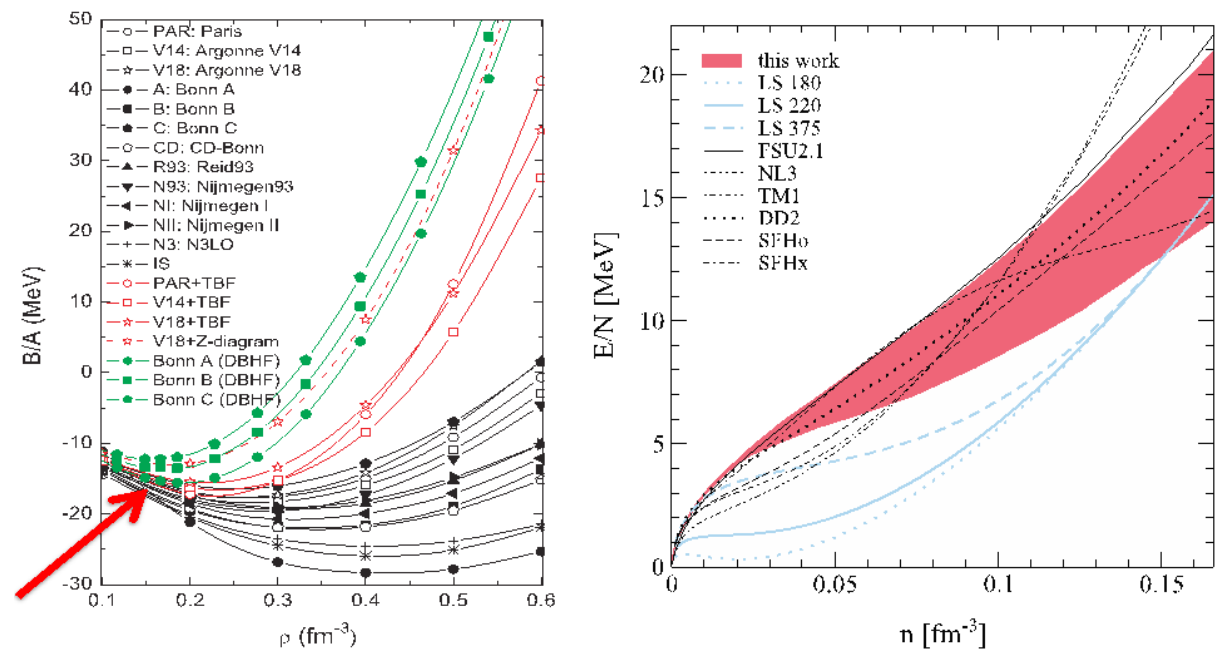

Figure 3. Left panel: Energy per nucleon of SNM as a function of particle number density obtained with different potentials and theoretical approaches. Taken from [8]. Right panel: Energy per particle of pure neutron matter at sub-saturation particle number density as calculated in Chiral Effective Field Theory (CEFT) and compared with selected EoS. For details see Ref. 9. Note a difference in notation for energy per particle (y-axis) and particle number densities (x-axis) used for the same quantities in Refs. 8 and 9. 
nucleon-nucleon $(\mathrm{N}-\mathrm{N})$ interaction and include the effect of the medium using techniques such as Bruckner-Hartree-Fock (BHF) and Dirac-Bruckner-Hartree-Fock (DBHF). As illustrated in Figure 3 (left panel), these basic quantities fail to be reproduced well by a variety of N-N interactions in the frequently used BHF approach (black curves). Only slightly better, but still poor, performance is found when three-body forces (TBF) are added (red curves). This addition increases uncertainty in the models, as TBF are not well known and contain additional variable parameters. The DBHF yield better results (green curves) but still lack accuracy.

Right panel of Figure 3 demonstrates results of the calculation of binding energy per particle in an even simpler system, pure neutron matter at sub-saturation density. The modern CEFT approach in the $\mathrm{N}^{3} \mathrm{LO}$ order yields the energy per particle as a function of the particle number density with the uncertainty depicted by the red (shaded) area. This result is compared with predictions of a selected set of EoS [9]. It is clear that even in this very simple case of nuclear matter there is a need to find stronger constraints on the EoS.

Even more uncertainty is found in attempts to generate the EoS of quark matter, expected to appear at high densities following a chiral hadron-quark phase transition. It is not possible at present to predict the conditions (baryonic density and temperature) at which such a transition would occur. We illustrate some EoS of quark matter constructed using the Dyson-Schwinger [10], Chromo-Dielectric [11], Nambu-Jona-Lasinio [11], perturbative QCD [12] and MIT bag [13] models in Figure 4.

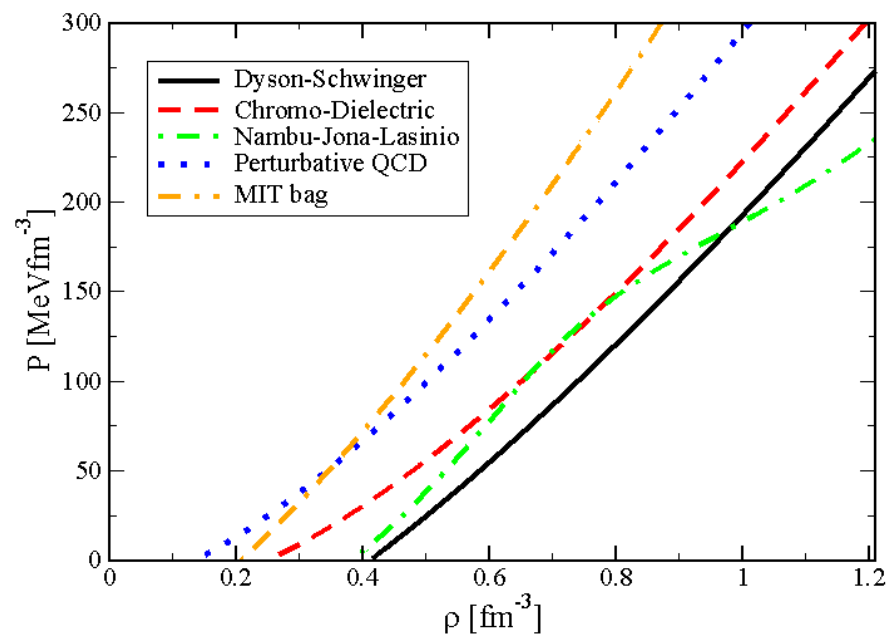

Figure 4. Equations of state of quark matter, as predicted by selected models [10 - 13].

The lack of adequate constraints on theoretical prediction of the EoS led to efforts to construct a hybrid empirical EoS, made up of components from theory, astrophysical observation and terrestrial data. We will discuss these components more fully in Section 3. Here we mention one such example presented by Steiner et al. [14] and shown in Figure 5. The authors used their analysis of recent observations of both transiently accreting and bursting X-ray sources and determined the $68 \%$ and $95 \%$ confidence intervals of the EOS of dense matter, with the estimated uncertainty of the pressure approximately $30 \%-50 \%$ at all densities achievable in neutron star interiors. This data have been combined with contributions to the EoS coming from analysis of heavy-ion collision experiments at lower densities and finally augmented by theoretical predictions of effective-field theory (EFT) and Quantum-MonteCarlo models at low densities and pressures.

Such an approach lacks internal consistency from a microscopic point of view. Its practical value both as an EoS to be used in applications and as a constraint on more physics based EoS is limited since at any number density the predicted pressure range is very wide. 


\section{Observational constraints on high-density EoS.}

Discussion in the previous section leads to the obvious question whether it is at all feasible to constrain the variety of models and their numerous parameters from available data. The question can also be re-phrased to make the point that it is important to assess the sensitivity of available data to the microphysics of models. We concentrate on the basic parameters of cold neutron stars:

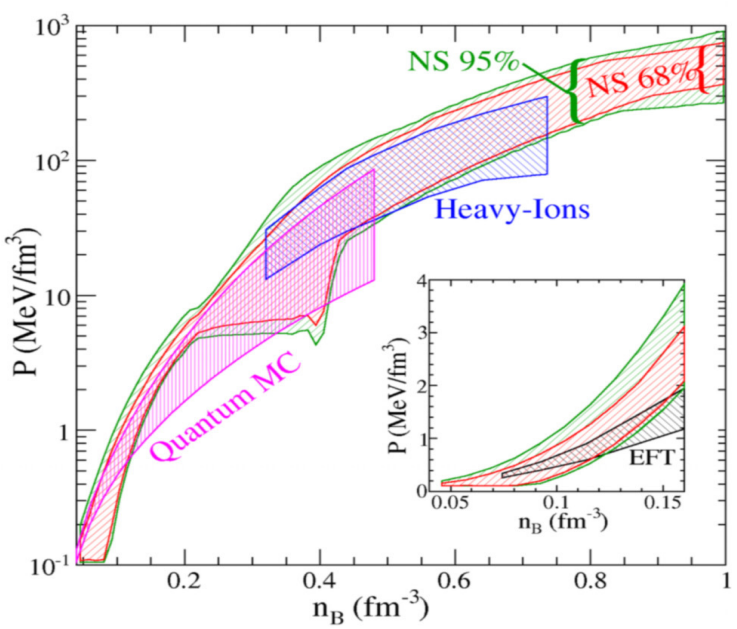

Figure 5. Empirical pressure dependence on baryon number density of high-density matter as presented in Ref. 14. For more details see text.

gravitational mass, simultaneous mass and radius information and cooling properties. Other astronomical phenomena, including core-collapse supernovae, are less suitable for this purpose because the mechanism of the collapse and the subsequent explosion, observed in nature, are not yet well understood and the possibility of testing the many EoS models is severely limited due to demands on computing time.

\subsection{Gravitational mass of a cold neutron star.}

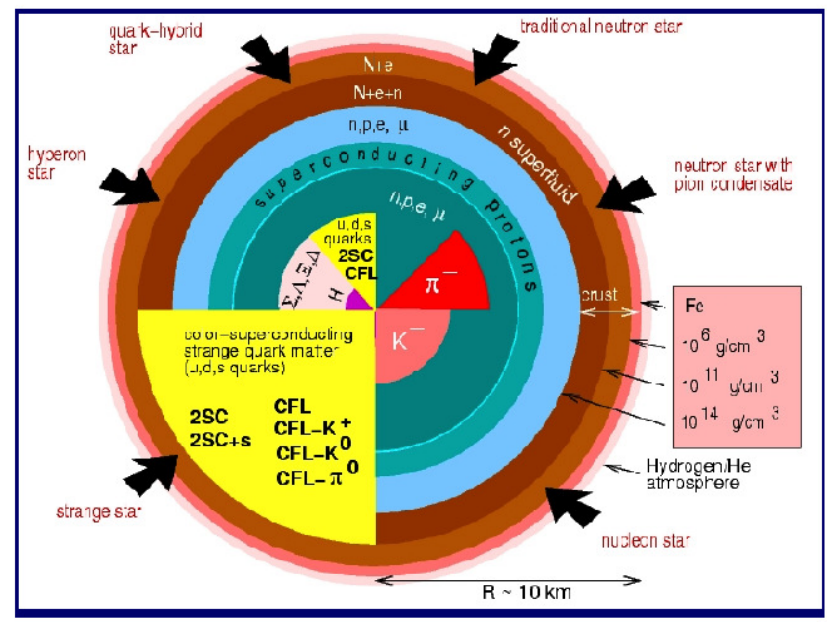

Figure 6. Proposed structure of a cold neutron star. Taken from Ref. 1. 
The exotic conditions believed to exist in cold neutron star allow development of many models of their interior based on a wide range of macro- and micro-physics. Considering the suggested composition of a neutron star, depicted in Figure 6, it is clear that successful modeling is a formidable task.

Gravitational mass and radius of a static neutron star can be obtained from the Tolman Oppenheimer - Volkoff (TOV) equations for hydrostatic equilibrium of a spherical object with isotropic density distribution in general relativity:

$$
\begin{aligned}
& \frac{d P}{d r}=-\frac{G M(r) \varepsilon}{r^{2}} \frac{\left(1+P / \varepsilon c^{2}\right)\left(1+4 \pi r^{3} P / M(r) c^{2}\right)}{1-2 G M(r) / r c^{2}} \\
& M(r)=\int_{0}^{r} 4 \pi r^{2} \varepsilon\left(r^{2}\right) d r
\end{aligned}
$$

Solution of these coupled equations yields sequences of neutron star models with a range of values for the central density $\varepsilon$. Integration of Equations 2, for any specified central density, gives directly the corresponding values for the total gravitational mass $M$ and radius $R$ of the star (the surface being at the location where the pressure vanishes).

The two things are important here. First, obviously the dependence of pressure $\mathrm{P}$ on central density $\varepsilon$ - the EoS - is required input to the TOV equations, bringing in the microphysics. Second, the output only yields gravitational mass as a function of a corresponding radius. This is a source of difficulty in relating observation to theory because data on mass and radius on the same star is very hard to obtain with high enough accuracy. It follows that even very accurate determinations of neutron star mass, currently available and summarized in Table 1, is a necessary but not a sufficient constraint for selection of realistic EoS.

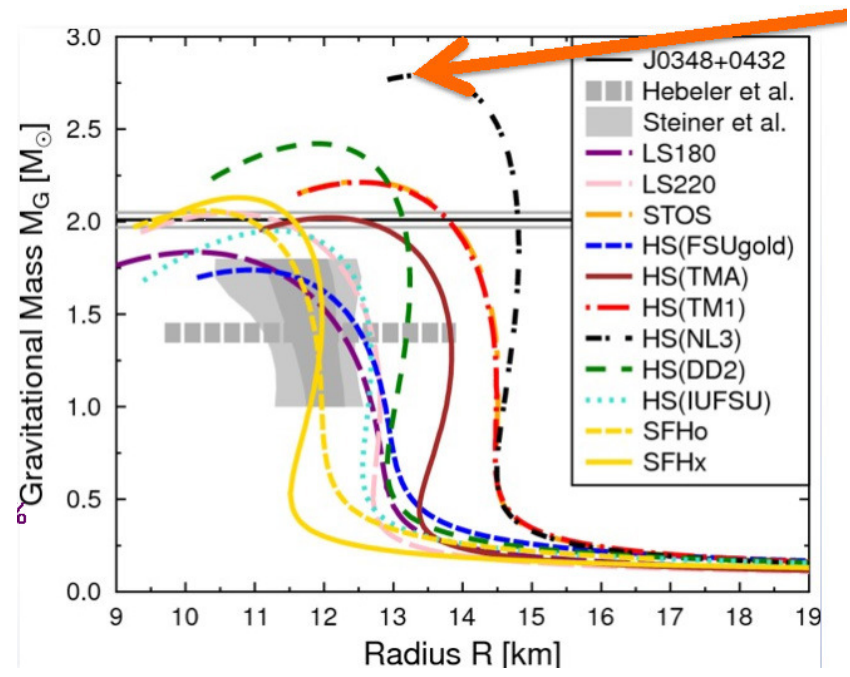

Figure 7. Examples of solutions of the TOV equations for different EoS. The arrow points to the location maximum mass model in one of the curves as an example. The largest mass yet reported is also shown. Taken from Ref. 15.

This situation is illustrated in Figure 7. The largest observed mass serves to eliminate some EoS, others satisfy this requirement and it is not possible to choose between them. 


\subsection{Simultaneous determination of mass and radius of a neutron star}

The main methods used in determination of neutron star mass and radius can be obtained from data on transient low mass X-ray binaries (LMXB) on X-ray photospheric radius expansion in Type-I X-ray bursts [21-25,29] or surface thermal emissions emission spectra from stars in quiescent LMXB (see e.g. $[24,26-28,30,31])$. The distance of the star is also required. It can be relatively well estimated for

Table 1. Examples of the most accurately measured neutron star masses.

\begin{tabular}{|l|l|l|l|}
\hline Pulsar & $\mathrm{M} / \mathrm{M}_{\odot}$ & error & Reference \\
\hline PSR J0737-3039 & 1.249 & 0.001 & Lyne et al. [16] \\
\hline PSR B1913+16 & 1.4414 & 0.0002 & Huyse and Taylor [17] \\
\hline PSR J1903+0327 & 1.667 & 0.021 & Freire et al. [18] \\
\hline PSR J1614-2230 & 1.97 & 0.04 & Demorest et al. [19] \\
\hline PSR J0348+0432 & 2.03 & 0.03 & Antoniades et al. [20] \\
\hline
\end{tabular}

Table 2. Selected estimates of mass and radius from data on Type-I X-ray bursts in transient LMXB and thermal emission from neutron star surface in quiescent LMXB. For more details about the method of analysis and observational data see references.

\begin{tabular}{|c|c|c|c|c|}
\hline Radius [km] & $\mathrm{M} / \mathrm{M}_{\odot}$ & $\begin{array}{c}\text { Conf } \\
\text { level \% }\end{array}$ & Sources & Reference \\
\hline $11-12$ & 1.4 & & $\begin{array}{l}\text { EXO 1745-248 } \\
\text { 4U 1608-522 } \\
\text { 4U 1820-30 } \\
\text { X7 (47 Tuc) } \\
\omega \text { Cen } \\
\text { M13 }\end{array}$ & Steiner et al. 2010 [24] \\
\hline$>14$ & $<2.3$ & & 4U 1724-307 & Suleimanov et al. $2011[25]$ \\
\hline $10.4-12.9$ & 1.4 & $\begin{array}{c}68,95 \\
\text { see text }\end{array}$ & $\begin{array}{l}\text { 4U 1608-522 } \\
\text { KS 1731-260 } \\
\text { EXO1745-248 } \\
\text { 4U 1820-30 } \\
\text { X7 (47 Tuc) } \\
\omega \text { Cen } \\
\text { M13 } \\
\text { NGC } 6397\end{array}$ & Steiner et al. 2013 [14] \\
\hline $7.6-10.4$ & $\begin{array}{c}\text { Mass } \\
\text { independent }\end{array}$ & 90 & $\begin{array}{l}\omega \text { Cen } \\
\text { M13 } \\
\text { NGC } 6397 \\
\text { NGC } 6304 \\
\text { M28 }\end{array}$ & Guillot et al. 2013 [27] \\
\hline $\begin{array}{l}11.4-12.8 \\
10.9-12.7\end{array}$ & $\begin{array}{c}1.4 \\
1.2-2.0\end{array}$ & 90 & $\begin{array}{l}\omega \text { Cen } \\
\text { M13 } \\
\text { NGC } 6397 \\
\text { NGC } 6304 \\
\text { M28 }\end{array}$ & Lattimer and Steiner 2013 [26] \\
\hline $\begin{array}{l}11.15-12.66 \\
10.45-12.45\end{array}$ & $\begin{array}{c}1.4 \\
\text { Basic EoS } \\
\text { Exo EoS }\end{array}$ & 90 & $\begin{array}{l}\omega \text { Cen } \\
\text { M13 } \\
\text { NGC } 6397 \\
\text { NGC } 6304 \\
\text { M28 }\end{array}$ & Lattimer and Steiner 2014 [28] \\
\hline$>13$ & $1.2-2.4$ & & $4 \mathrm{U} 1608-52$ & Poutanen et al. 2014 [29] \\
\hline $10-13$ & $\begin{array}{l}\text { Typical } \\
\text { masses }\end{array}$ & & $\begin{array}{l}\omega \text { Cen } \\
\text { NGC } 6397\end{array}$ & Heinke et al. 2014 [30] \\
\hline $9-14$ & $\begin{array}{c}1.4 \\
>1.8 \\
(1.4) \\
\end{array}$ & & $\begin{array}{l}\text { X5 (47 Tuc) } \\
\text { X7 (47 Tuc) }\end{array}$ & Heinke et al. 2014 [31] \\
\hline
\end{tabular}


stars in globular clusters, however, restriction to clusters limits the number of possible targets. Alternatively, it has to be inferred indirectly but the error of the results is significantly increased.

The data analysis is rather involved [21] and new effects, needed to be considered, are being discovered. Recent spectral measurement of Type-I X-ray bursts [29] revealed a strong dependence of the burst properties on the flux and spectral hardness of the persistent emission before burst which affects the extracted mass-radius information. The uncertainty as to effects of the composition of the stellar atmosphere and the any absorbing material along the line of sight is one of the main reasons for the differences in the results, reported by different groups based, in some cases, on the same observational data (see Table 2).

It is clear that much work is needed before such mass-radius data can form a reliable constraint on the EoS of neutron stars.

\subsection{Neutron star cooling.}

Interesting data from the Chandra X-ray observatory, reported by Ho and Heinke [32,33] suggested unusually rapid cooling of an isolated neutron star with carbon atmosphere and low magnetic field in Cassiopeia A (Cas A) - the remnant of the historical 1680 core-collapse supernova. They found the rate of cooling to be significantly larger that that expected from the modified (or medium modified) URCA process. This challenging observation prompted several theoretical interpretations having different physical bases: (i) the "minimal cooling paradigm" [34], (ii) the "nuclear medium cooling scenario" [35] and general relativistic effects [36] and no particular assumptions on microscopic structure of the star [37].

Theory (i) is based on the assumption that the cooling is enhanced by neutrino emission following the onset of breaking and formation of neutron Cooper pairs in the ${ }^{3} \mathrm{P}_{2}$ channel in the star's core. The model assumes that the star is already in a proton superconducting state. The rapidity of the cooling is presented as firm evidence for the presence of neutron superfluidity and proton superconductivity in the core of the neutron star. Theory (ii) goes beyond the minimal cooling model and introduces medium-modified one-pion exchange in dense matter and polarization effects in the pair-breaking of neutrons and protons.

The problem with models (i) and (ii) is that the mass of the neutron star in Cas A is not certain and it is not clear whether nucleons can exists in its core or whether they make transition to hyperonic or quark matter. Ho and Heinke [32,33] estimated $\mathrm{M} \approx 1.5-2.4 \mathrm{M}_{\odot}, \mathrm{R} \approx 8-18 \mathrm{~km}$. If the mass is larger than about $1.4 \mathrm{M}_{\odot}$, it is unlikely that nucleons exist in the core and the proposition that the core makes transition to a superfluid neutron state becomes untenable.

Theory (iii) approaches the rapid cooling from a very different point of view. Negreiros et al [36] developed a model of thermal evolution of rotationally deformed neutron stars in two dimensions in the framework of general relativity. They showed that the observational data could be reproduced to the same level of accuracy as is obtained by theories based only on neutron and proton superfluidity. However, they have to assume that Cas A is rapidly rotating.

Theory (iv) developed by Yakovlev at al [37] studied cooling of Cas A without a specific assumption on microscopic structure of the star and got results compatible with observation.

Very recently the report of Ho and Heinke [32] has been seriously challenged. Posselt et al. [38] reported two dedicated observations with an optimal observational setup, carried out in 2006 and 2012, which did not show statistically significant temperature drop, although, at the $3 \sigma$ level, the rapid cooling could not be completely ruled out. Posselt et al. concluded "Overall, our results (20062012) are consistent with no temperature decline at all, or a smaller temperature decline than that reported for the data suffering from pile-up and acquired in Graded mode during the time interval 2000-2012. A longer time base of data with negligible pile-up and a better knowledge of the ACIS filter contamination changes are needed to assess any temperature or flux change with higher certainty". To firmly exclude or confirm the rapid cooling and establish a tight constraint on 
the cooling rate, they proposed an additional dedicated observation of this object, undistorted by the systematic errors (Chandra proposal ID \#16500703) [39].

We demonstrate the results in Figure 6. This figure includes data by Elshamouty et al., [40] who added a 2012 point and re-analyzed data from all Chandra detectors (only ACIS data were included in [32]). The vertical shift between data [32] and [40] is due to different normalization. The full caption taken from [38] is shown to help the reader to appreciate the data.

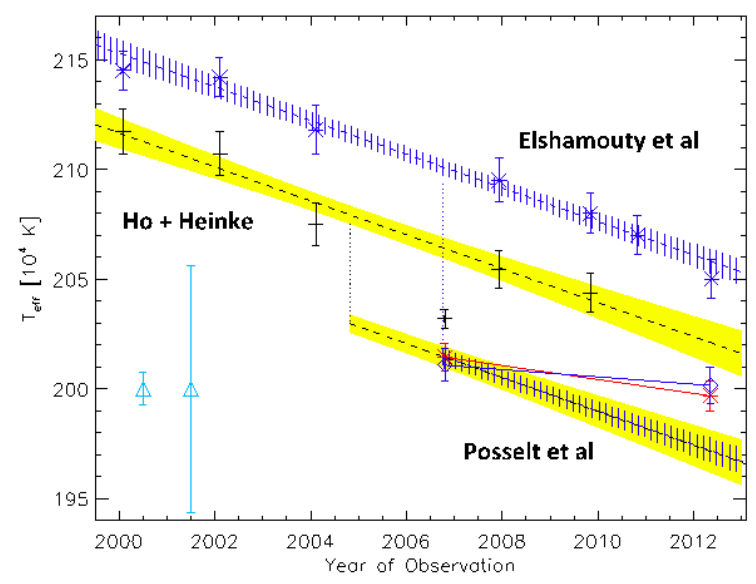

Figure 6. Temperature change over time. The black crosses and blue asterisks mark the temperatures and their $1 \sigma$ errors as reported in Ref. 32 (their Table 1 and Figure 2) and (their Table 2), respectively. Their fit results were derived from piled data telemetered in Graded mode, using carbon atmosphere models $(\mathrm{MNS}=1.648 \mathrm{M} \odot$ and RNS $=10.3 \mathrm{~km}[32,33] ; \mathrm{MNS}=1.62 \mathrm{M} \odot$ and $\mathrm{RNS}=10.19 \mathrm{~km} ;[40)]$ with the same fixed $\mathrm{NH}$ for all observations. The black dashed line and the yellow area indicate the results of a linear regression fit and its $1 \sigma$ error to the $[32,33]$ data points if we choose the average of their observing epochs as reference time, $\mathrm{t}_{0}=2004.82$ (dotted vertical black line). The blue dashed line and the blue-striped area indicate the results of a linear regression fit and its $1 \sigma$ error to the [40] data points if we choose the average of their observing epochs as reference time, $\mathrm{t}_{0}=2006.75$ [36] (dotted vertical blue line). The [38] fit results from Table 1 for a carbon atmosphere model with similar gravitational parameters $(\mathrm{MNS}=1.647 \mathrm{M} \odot$ and $\mathrm{RNS}=10.33 \mathrm{~km})$ are marked with red star points (same NH in both epochs) and blue, diamond points (different NH in 2006 and 2012). For completeness, the 2006 temperature by [32,33] is shown. All errors in this plot are $1 \sigma$ errors. In the lower left corner, a typical $1 \sigma$ temperature uncertainty for the cases of fixed (left) and free, but tied (right) normalizations is shown. See text for a detailed discussion. Taken from Ref. 38.

The existence of several different theories explaining the rapid cooling of Cas A equally well implies that this data alone cannot distinguish between them. The mass of Cas A (which is unlikely to be determined with any certainty as Cas A is an isolated star) would be required with good accuracy to confirm or exclude the neutron superfluidity theory and the period of rotation would be needed to justify application of the relativistic theory of cooling to this neutron star. What is more disturbing is that, if the results of Posselt et al. [38] should prove correct, theories (i) - (iv) have merely shown the ability to reproduce spurious data.

\section{Terrestrial constraints}

\subsection{Heavy-ion collisions}

Heavy-ion collisions (HIC) are the only terrestrial events in which hot high density matter is created. The beam energies range from $\sim 35 \mathrm{MeV} / \mathrm{A}$ to $5 \mathrm{TeV} / \mathrm{A}$ at different facilities, NSCL, Texas A\&M, GSI, RHIC and LHC (existing), and FAIR and NICA (future). Au and Sn projectiles are frequently used and elliptical and transverse particle flow is usually detected. 
At beam energies $\sim 35 \mathrm{MeV} / \mathrm{A}-2 \mathrm{GeV} / \mathrm{A}$, which access the region below nuclear density, and temperatures up to several tens of $\mathrm{MeV}$, neutron/proton spectral ratios and neutron, hydrogen and light fragment flows can been studied [41]. One of the important results of such experiments is information on the nuclear symmetry energy and its density dependence. The data are used to fit parameters of transport models, which provide energy density and pressure in the matter, i.e. the EoS [42-46]. The much-discussed question arises whether such EoS can be meaningfully used to constrain the EoS of high-density matter in neutron stars and supernovae.

Matter created at higher beam energies has typically very low particle number density (significantly lower than the saturation density of the symmetric nuclear matter, $0.16 \mathrm{fm}^{-3}$ ), but relatively high energy density $\left(1-6 \mathrm{GeV} / \mathrm{fm}^{3}\right.$ dependent on beam energy), close to zero baryon chemical potential. The matter is heated to several hundred of $\mathrm{MeV}$. The time-scale between the collision and the chemical and thermal freeze-out is of order $10^{-23}$ to-24 $\mathrm{s}$ which implies that the creation of particles during the collision is governed only by the strong interaction. This has the important consequence that only processes that conserve strangeness and the baryon and lepton number can occur. Analysis of particle spectra and ratios indicate that quark-gluon plasma (QGP) may be reached and quark-antiquark pairs, strange baryons and mesons and pions and a significant number of their antiparticles are subsequently created. The matter is lepton poor, in particular containing very few neutrinos.

At medium beam energies used in experiments with $\mathrm{Au}$ or Sn projectiles inelastic (N-N) scattering becomes significant and matter consists mostly of $\mathrm{N}, \mathrm{N}^{*}, \Delta$, light nuclear fragments such as ${ }^{3} \mathrm{H},{ }^{3} \mathrm{He},{ }^{7} \mathrm{Li},{ }^{7} \mathrm{Be}$, and pions. Strangeness becomes less important, but kaons may be expected [46]. It is claimed that, for a small fraction of the time, number densities as high as 5 times nuclear saturation density $[41,43,44]$ can be achieved in ${ }^{197} \mathrm{Au}+{ }^{197} \mathrm{Au}$ collisions at beam energies $\sim 50-100 \mathrm{MeV} / \mathrm{A}$, due to inertial confinement. The matter cools fast from about an initial $\mathrm{T} \sim 50 \mathrm{MeV}$ and dilutes to subnuclear densities.

Theoretical approaches to the dynamics of $\mathrm{HIC}$ at medium energies are either algebraic, based on self-consistent solution of the Boltzmann equations used in transport models (see e.g. [42-45]), or geometric, using Molecular Dynamics simulation (MD) in which particles are treated as Gaussian wave packets in a unit cell [47-49]. Both classes of theories yield predictions on trajectories of particle emitted from the collision area. In addition, the MD models provide information on emission of clusters.

Without going into details of application of these models of the collision to actual data, there are several open questions in interpretation of the results and their implications for understanding of the structure of high-density matter. Is obvious that constraints extracted from HIC experiments are not applicable to matter in cold neutron stars and core-collapse supernovae. The composition of the matter in these astrophysical objects is very different from that generated in HIC collisions and the EoS will be different manifestations of the "universal" EoS. To make this important point clear, we contrast basic properties of the two scenarios in Table 3.

\subsection{Hypernuclei}

Realistic models of neutron star matter need to include hyperons at central densities exceeding 2 3 times nuclear saturation density. Then it is important to model as well as possible hyperon-nucleon and hyperon-hyperon forces, acting in the nuclear environment.

The study of $\Lambda$ hypernuclei has a long history, with the shell structure mapped out across the periodic table [50-55]. Systematic studies of the energy levels of light $\Lambda$ hypernuclei have enabled the extraction of considerable detail concerning the effective $\Lambda-\mathrm{N}$ interaction. Studies of electroproduction of hypernuclei at JLab may provide important new information in this area.

As for $\Sigma$ and $\Xi$ hypernuclei, the situation is quite different. The special case of ${ }^{4} \mathrm{He}$ aside, there is no experimental evidence for any $\Sigma$ hypernuclei [56-58], despite extensive searches. Indeed, it seems likely that the $\Sigma$-nucleus interaction is somewhat repulsive and that there are no bound $\Sigma$ hypernuclei beyond $A=4$. In the case of the $\Xi$, the experimental situation is very challenging, with just a handful 
of observations of doubly strange nuclei. Long awaited studies of $\Xi$ hypernuclei with new facilities at J-PARC and FAIR, may show these hypernuclei exist. It would be helpful to have predictions from various theoretical models, however no firm conclusions can be drawn at present.

Table 3. Comparison of (a) basic property of matter created in HIC with beam energies $<1 \mathrm{GeV} / \mathrm{A}$ and (b) matter in proto-neutron stars (PNS) for masses of progenitor stars 8 - 20 solar mass. S, B, L stands for strangeness, baryon and lepton number, respectively. $\mathrm{N}$ and $\mathrm{N}^{*}$ are $\mathrm{N}$ - baryons (nucleons) and their resonance, $\Delta, \Delta^{*}$ are $\Delta$ - baryons and their resonances.

\begin{tabular}{|l|l|l|}
\hline & \multicolumn{1}{|c|}{$\mathrm{HIC}$} & \multicolumn{1}{c|}{ PNS } \\
\hline Temperature & $<50 \mathrm{MeV}$ & $<50 \mathrm{MeV}$ \\
\hline Energy density $\varepsilon$ & $\sim 1-2 \mathrm{GeV} / \mathrm{fm}^{3}$ & $\sim 1 \mathrm{GeV} / \mathrm{fm}^{3}$ \\
\hline Baryon density $\rho$ & $<1.5 \rho_{0}$ & $2-3 \rho_{0}$ \\
\hline Time scale to cool down & $10^{-23-24} \mathrm{~s}$ & $\begin{array}{l}1-10 \mathrm{~s} \\
\rho \text { and T dependent }\end{array}$ \\
& & $\begin{array}{l}\text { Strong and Weak } \\
\mathrm{B}, \mathrm{L} \text { conserved }\end{array}$ \\
\hline Interaction & Strong & $\mathrm{n}, \mathrm{p}$ \\
\hline Composition: & $\mathrm{S}, \mathrm{B}, \mathrm{L}$ conserved \\
\hline & $\pi, \Delta, \Delta^{*}$ & no pions \\
\hline & strangeness rare & strange baryons and mesons \\
\hline & no leptons & copious leptons \\
\hline Symmetry & symmetric & highly asymmetric \\
\hline Thermal equilibrium & \multicolumn{1}{c|}{ yes } \\
\hline
\end{tabular}

\subsection{Lattice QCD}

It would be desirable to test predictions of many theories against results obtained in lattice QCD, which can be regarded as experimental data [2]. However, only calculation at zero baryo-chemical potential is available at present. Because of the well-known numerical sign problem [59], it is questionable whether the calculation can be ever extended to finite chemical potentials. The use of physical quark and pion masses is outside reach at present because of limitations of currently available computation and interpolation methods have to be used. Although there is some hope that lattice QCD will eventually provide data directly aiding determination of the EoS of high-density matter, its realization is not to be expected in the near future.

\section{Quark Meson Coupling (QMC) model.}

It has become apparent in previous sections that currently available observational and experimental data are not yet adequate to uniquely constrain the EoS of high-density matter. We suggest that, along with the need for more precise data, a principle aim for theorists should be to develop new and improved theories in which parameters are directly related to microphysical quantities for which good estimates can be made. In this section we bring an example of a theory of this type, which has not hitherto received much attention.

Conceptually, one of the main problems in the nuclear many-body problem is to incorporate medium effects into the calculation. In contrast to, for example, elementary QED, where the force between two electric charges is quantified by the Coulomb law and forces among many electrons can be calculated precisely using the principle of superposition, no equivalent approach exists in nuclear physics. Scattering experiments with free nucleons provide information about free N-N potentials, but a complicated numerical treatment is necessary to transform these potentials to a form describing adequately the saturation of forces among nucleons in the nuclear environment. The most microscopic 
methods start from a realistic two-body free N-N interaction with parameters fitted to N-N scattering phase shifts in different partial wave channels and to properties of the deuteron [60]. By taking these bare N-N interactions as input into a many-body formalism, such as the relativistic Dirac-BrucknerHartree-Fock (DBHF) approximation and its nonrelativistic counterpart BHF [61,62], variational methods [63,64], fully self-consistent treatment of short-range correlations [65], self-consistent Green's function (SCGF) models [66], quantum Monte Carlo techniques [67-72] and chiral effective field theory $[9,73,74]$, an effective $\mathrm{N}-\mathrm{N}$ interaction, which includes the effect of the medium, is derived and the many-body problem is approximately solved. However, this treatment introduces a numbers of parameters without transparent physical meaning.

The idea of modeling the N-N interaction using quark degrees of freedom originated in the late 1980's with Guichon [75] and Guichon and Thomas [76] who suggested that the origin of nuclear many body forces and of the saturation of nuclear forces can be found in the modification of the structure of a nucleon immersed in a meson field generated by the surrounding nucleons. The model has been adopted and developed in different forms, in Australia, Japan, China, Korea, Brazil and Europe (see for more details [77]). We use here the version of the model described in [5].

The basic assumption is that instead of the usual treatment of modeling nuclear forces through exchange of mesons coupled to nucleons, taken as point-like particles, this exchange takes place directly between quarks in different non-overlapping nucleons, taken as having a structure in the form, for example, of the MIT bag. It is the mutual interaction between the quark and meson fields, which leads to the saturation property of nuclear forces [75].
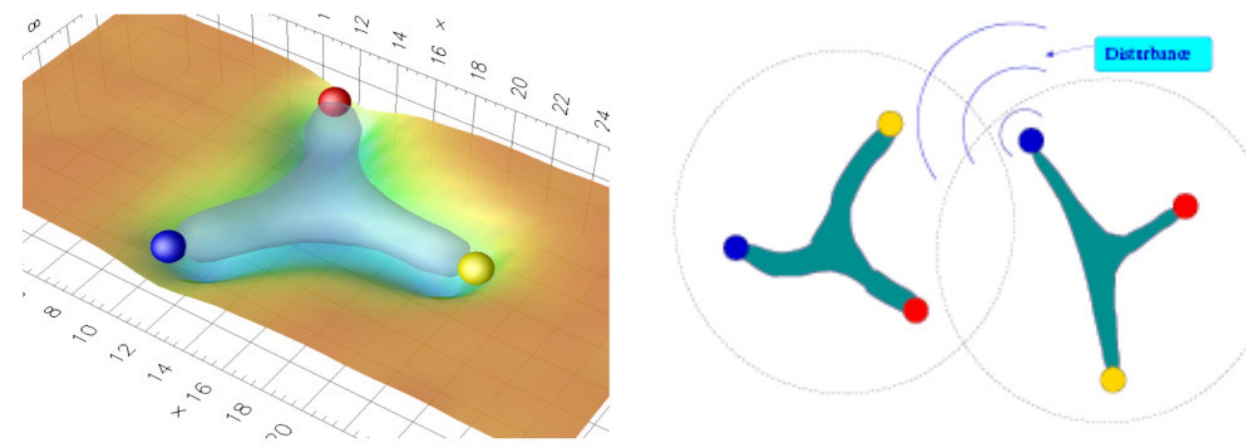

Figure 7. Left panel: Surface plot of the correlation function in lattice QCD for Y-shape source with quark positions depicted by colour spheres [79]. The formation of gluon flux-tubes is shown. Right panel: cartoon of interaction of two nucleons through fluctuation, described by meson fields, in QCD vacuum, (by P. A. M. Guichon) .

The bag model is taken as an effective realization of confinement, which must not be taken too literally. The QCD lattice simulations of Bissey et al. [78,79] indicate that the true confinement picture is closer to a Y-shaped color string attached to the quarks (see Figure 7). Outside this relatively thin string one has the ordinary, non-perturbative vacuum, where the quarks from other hadrons can pass without disturbing the structure very much. So, even though the bag model imposes a strict condition, which prevents the quarks from travelling through its boundary, this must be seen as the average representation of a more complex situation and one should not attribute a deep physical meaning to the surface of the cavity nor to its size. In particular, estimating the density at which the QMC approximation breaks down as the reciprocal of the bag volume is certainly too pessimistic [5]. Of course, there is a density above which such models become inadequate but it is safe to assume that this critical density is large enough that we can use the model to predict the properties of neutron stars. 
All variants of the QMC model, outlined briefly below, rather than starting with the bare N-N force, begin with the concept of a nucleon, modeled by the MIT bag, immersed in a nuclear medium. The effects of the coupling to the $u$ and $d$ quarks of a scalar-isoscalar meson $(\sigma)$ mean field, generated by all the other nucleons in the medium, on the internal structure of that nucleon are then included self-consistently. As in earlier boson-exchange models, the $\sigma$ is a crude but convenient way to simulate the effects of correlated two-pion exchange between hadrons. The quarks are also coupled to $\omega$ and $\rho$ mesons, which, at least at the Hartree level, simply shift quark energies.

Assuming [75] that the nucleon is located in average, time independent, meson fields $\bar{\sigma}, \bar{\omega}$ and $\bar{\rho}$, the MIT bag equations are solved to obtain the expression for the quark field, depending on the quarkmeson coupling constants $g_{\sigma}^{q}, g_{\sigma}^{q}$ and $g_{\rho}^{q}$, the free nucleon radius $\mathrm{R}_{0}$ and the vacuum energy density $\mathrm{B}$, adjusted to yield the mass of the free bag equal to the spin-isospin averaged mass of the nucleon and the $\Delta$. Of the coupling constants, only $g_{\sigma}^{q}$ is density dependent. These quark-meson couplings describe the interaction between quarks in different hadrons. They act as the source of mean fields in medium as well as serving to modify the equation of motion of the confined quarks. This leads to a self-consistency problem, which is highly nontrivial for the scalar field. As a result, the effective strength of the coupling of the scalar meson to a hadron containing light quarks is suppressed as the scalar field increases, or equivalently, as the density increases.

As a result of this self-consistent calculation at the quark level, one can express the in-medium nucleon masses $M_{e f f}(\bar{\sigma})$, given as a function of the scalar field, through a calculated, densitydependent, scalar coupling,

$$
M_{e f f}(\bar{\sigma})=M-g_{\sigma N} \bar{\sigma}+\frac{d}{2}\left(g_{\sigma N} \bar{\sigma}\right)^{2}
$$

where $d$ is the scalar polarizability of the nucleon. This scalar polarizability is an essential feature of the $\mathrm{QMC}$ model and is related to the bag radius $\mathrm{R}_{\mathrm{B}}$ as $d=0.0044+0.211 R_{B}-0.0357 R_{B}^{2} \mathrm{~A}$ simple relation relates the quark-meson coupling constants to the nucleon-meson constants

$$
g_{\sigma N}=3 g_{\sigma}^{q} \int_{B \times g} d \vec{r} \bar{q} q(\vec{r}) \quad \mathrm{g}_{\sigma N}=3 g_{\omega}^{q} \quad \mathrm{~g}_{\rho N}=g_{\rho}^{q}
$$

where $q$ is the valence quark wavefunction for the MIT bag. The influence of nucleon substructure, in a mean-field approximation, is entirely described in terms of the parameterization of the effective mass of the nucleon through the density-dependent scalar coupling derived from the quark model of the nucleon and $g_{\sigma}^{q}$. Therefore the explicit description of the internal structure of the nucleons can be replaced by constructing an effective relativistic Lagrangian on the hadronic level, with the calculated nonlinear $\sigma$-nucleon couplings [75] and proceeding to solve the RMF equations in a standard way.

The scalar polarizability describes the self-consistent response to the applied mean scalar field which tends to oppose that applied field. By analogy with the electric polarizability of an atom, which tends to arrange its internal structure to oppose an applied electric field, the reduction of the $\sigma \mathrm{N}$ coupling, increasing $\sigma$ field is the origin of the explanation of saturation of nuclear forces in QMC model. The scalar polarizability is a calculated property of the nucleon and hence introduces no new parameters into the model. Moreover, it is this scalar polarizability, which yields the density dependence of the derived N-N effective force, or equivalently the three-body forces among nucleons.

The outline of the essential features of the QMC model has been presented so far for nucleons. However, it is directly applicable for any hadrons [75-77]. That is, the model predicts the existence and strength of the two- and three-body forces between not just nucleons, but nucleons and hyperons and hyperons and other hyperons, without additional parameters.

To summarize, the essential feature of the QMC model is that it depends essentially only on three adjustable parameters, the nucleon-meson coupling constants $g_{\sigma N}, g_{\omega N}$ and $g_{\rho N}$. It is customary to define 


$$
G_{\sigma}=\frac{g_{\sigma N}^{2}}{m_{\sigma}^{2}} \quad G_{\omega}=\frac{g_{\omega N}^{2}}{m_{\omega}^{2}} \quad G_{\rho}=\frac{g_{\rho N}^{2}}{m_{\rho}^{2}} .
$$

Their value is adjusted to reproduce the binding energy and the asymmetry energy coefficient of ordinary nuclear matter at the saturation point. The other parameters are the bag radius (constrained to lie between $0.8-1.0 \mathrm{fm}$ ) and masses of the mesons, which are taken to be the physical masses for $\omega$ and $\rho$. The $\sigma$ meson mass and its very existence as a particle is not completely clear as yet. It is usually identified with the $\pi \pi$ resonance which has a wide decay width, observed in scattering experiments. The best estimates of $\sigma$ meson mass lie between $600-700 \mathrm{MeV}$, consistent with applications in other areas (see e.g. [55, 94] and references therein).

All the other elements of the model are calculated within the model or determined by symmetry. Consistent with the requirement for improved theory, all the parameters of the QMC model have physical meaning and cannot be varied outside well-defined boundaries.

\subsection{The QMC model in nuclear matter and neutron stars.}

The performance of the QMC model as concerned the EoS of high-density matter and neutron stars has been studied in [5] (similar results have been obtained also with slightly variant version of the model in [77, 80-82]). We briefly review the main points of this work, comparing QMC700 (700 stands for the choice of the $\sigma$ meson mass equal to $700 \mathrm{MeV}$ ), with results from APR [63] and SkM* [83], chosen as widely used representatives of Skyrme and realistic potential models. APR is based on the $\mathrm{A} 18+\delta \mathrm{v}+\mathrm{UIX}^{*}$ which includes three-body forces (over 20 parameters) and SkM* is dependent on seven variable parameters.

Figure 8 shows the EoS of pure neutron matter and symmetric matter (left panel) for the QMC700 model compared with results for APR and $\mathrm{SkM}^{*}$ EoS (middle panel) and comparison of predictions of all three models for the density dependence of the symmetry energy (right panel). All models are adjusted to the saturation properties of SNM, although the PNM predictions differ considerably at higher density. The behaviour of the symmetry energy, similar at densities close to the saturation density, diverged dramatically at increased density. We note that the results of the QMC calculation, dependent only on three well-defined parameters, are comparable with other models, both widely used in the literature, based on very different physics and requiring many more parameters which have to be chosen with limited underlying physics guidance.
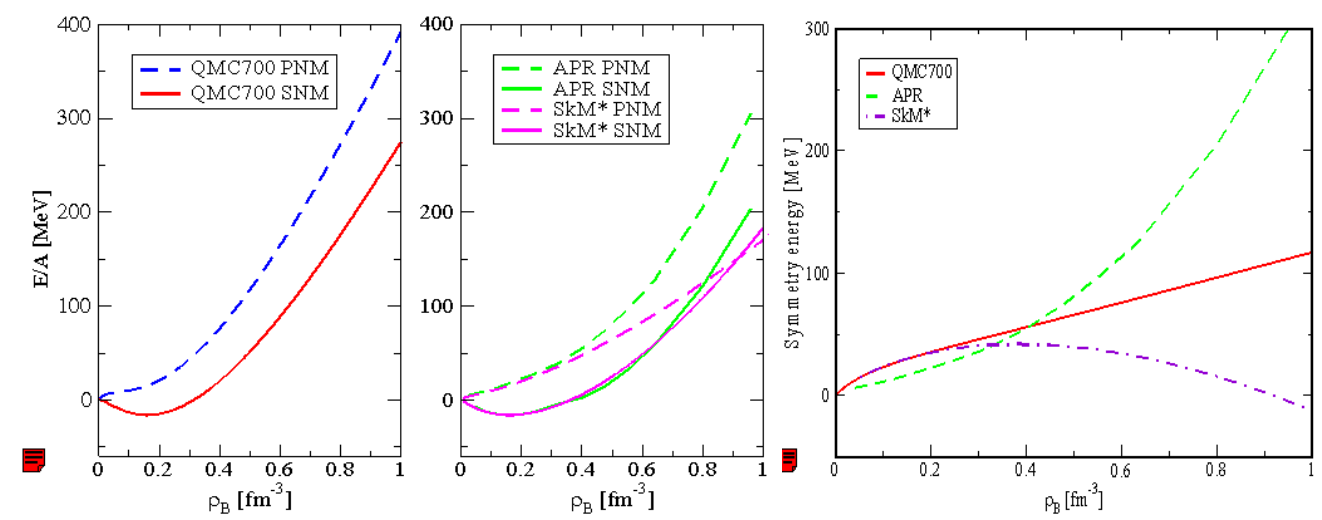

Figure 8. Equations of state of PNM and SNM as predicted by QMC700 model (left panel), in comparison with results for APR and SkM* EoS. The calculated density dependence of the symmetry energy for the three EoS is shown in the right panel.

One persistent feature of the QMC models is that they predict a value of the incompressibility of symmetric nuclear matter at saturation, $\mathrm{K}_{0}$, between about $280-350 \mathrm{MeV}$. This value is somewhat higher than commonly accepted. In the light of the recent re-analysis of data on giant monopole 
resonances which finds $250<\mathrm{K}_{0}<315 \mathrm{MeV}$ [85], the QMC predictions are acceptable. It affects the maximum mass of the neutron star only in a limited way. The incompressibility and composition of matter at densities expected in the core of a neutron star play an essential role.

The pressure dependence of the energy per particle (EoS), directly relevant for calculation of neutron stars, of $\beta$-equilibrium matter is presented in Figure 9. The left hand panel shows QMC700 results. The dashed curve would be followed by a version of QMC700 (N-QMC700) arbitrarily limited to contain only nucleons at all densities. The full curve is the prediction for the complete model including the full baryon octet (F-QMC700). In this model, the natural appearance of hyperons which occurs at $\varepsilon \sim 600 \mathrm{MeVfm}^{-3}$, as expected softens the EoS which, as we will see, is vitally important for the existence of heavier neutron stars. In the right panel, the APR and SkM* results (being nucleon-only) show no softening. We also show in this panel results of the Bethe-Johnson (BJ) model [84], which also contains hyperons. It shows behaviour broadly similar to F-QMC700, however there are differences of detail since, the composition and threshold densities of non-nucleon species in the BJ model are different from those obtained within the QMC model.
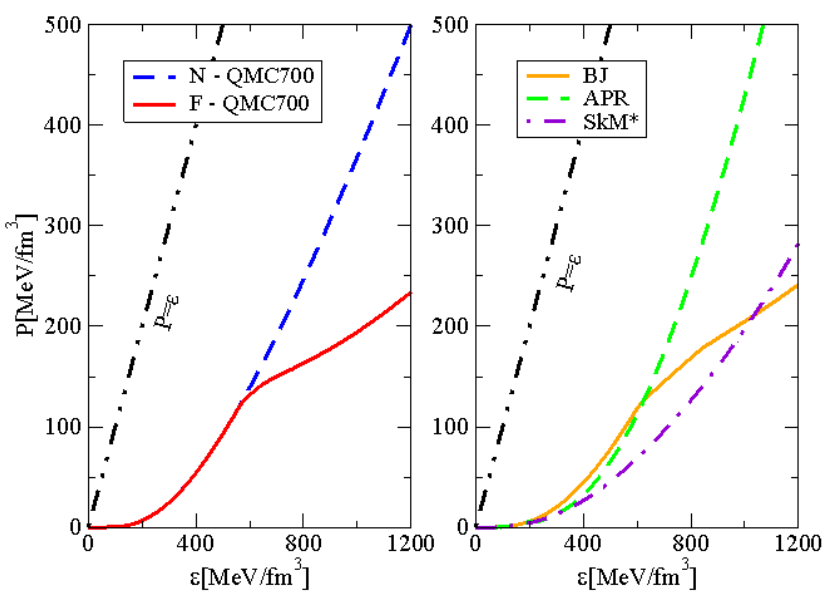

Figure 9. Left panel: Pressure as a function of total energy density for sample EoS for $\beta$-equilibrium matter containing only nucleons and leptons $\mathrm{p}+\mathrm{n}+\mathrm{e}+\mu$ (N-QMC700) and the full octet and leptons (F-QMC700). Right panel: Results of BJ, APR, SkM ${ }^{\star}$. The curve $\mathrm{P}=\varepsilon$ shows the stiffest possible EoS. For more details see text.

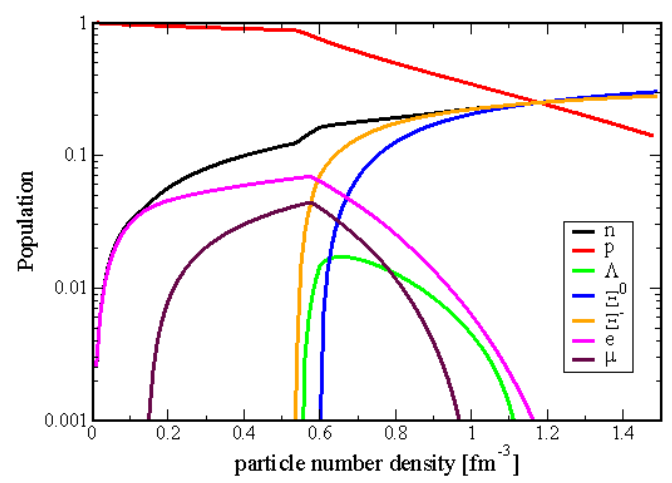

Figure 10. Relative population of baryons and leptons in $\beta$-equilibrium matter for QMC700. For more explanation see [5]. 
However, the mechanism of softening of EoS is complex and variation of the composition of the matter is not the only way to achieve it. The pressure also depends critically on the potentials acting between the particles present and, in turn, on the density dependence of the symmetry energy. Thus, for example, the SkM Skyrme model produces a very soft EoS, in contrast to APR. The predictions of the threshold densities for appearance of hyperons, essential for description of high-density and neutron stars, differ in the QMC model from the other RMF models. As shown in Figure 10, the QMC model predicts the onset of production of hyperons at densities above three times nuclear saturation density. The first hyperons to appear are the cascades, $\Xi$, together with the $\Lambda$ hyperon. The $\Sigma$ hyperons are not produced at densities below $1.2 \mathrm{fm}^{-3}$. The natural occurrence of hyperons is a direct consequence of features, which are present in the QMC model and absent in RMF (for details see [5]). The QMC result for $\Sigma$ hyperons is in line with the fact that no bound $\Sigma$-hypernucleus at medium
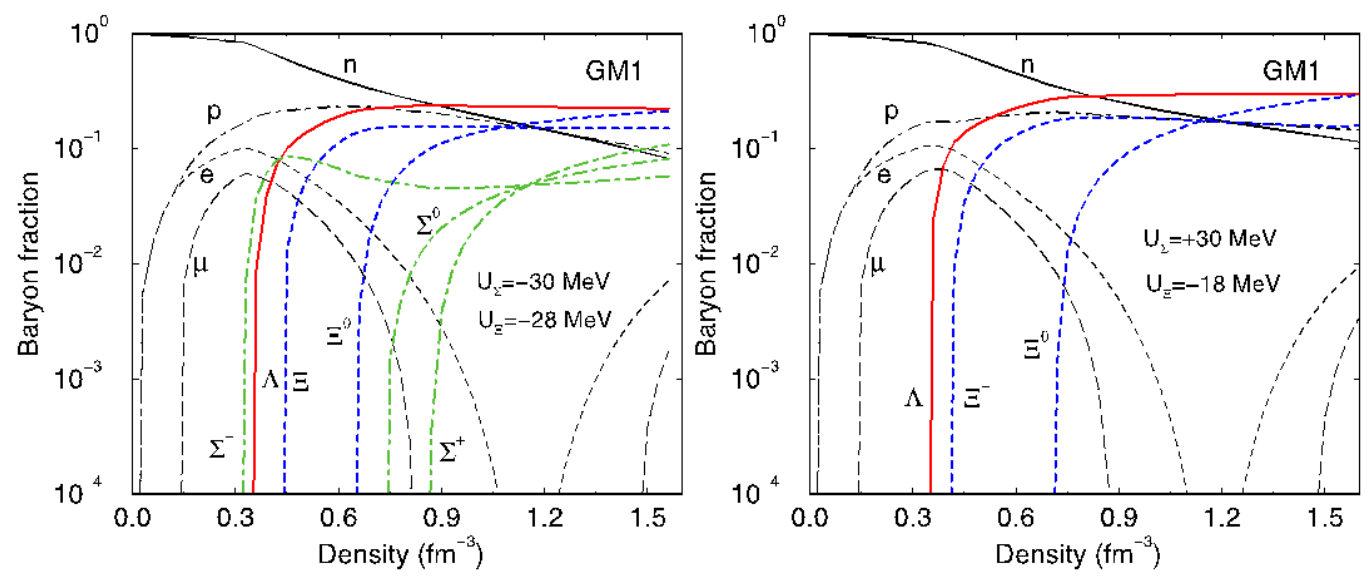

Figure 11. The composition of neutron star matter as a function of baryon densityin reltivisitc mean field model $[86,87]$. Hyperons appear around $2 \rho_{0}$. The presence of the $\Sigma$ hyperons depends crucially on the sign of the hyperon-nucleon potential, there are no $\Sigma$ hyperons present for a repulsive potential. Left plot: attractive $\Sigma$ potential, right plot: repulsive $\Sigma$ potential (see [87] for the details of the model used).

or high mass has been yet found despite dedicated search. The appearance of the $\Xi$ hyperons at densities lower than their predicted occurrence in standard RMF models may imply the existence of a bound $\Xi$-hypernucleus. Experimentally, this is still an open question, waiting for possible confirmation when J-PARC will start operation.

In the RMF models, the sequence of the appearance of hyperons with increasing density usually follows their masses, predicting $\Lambda$ hyperon at lowest density, followed by $\Sigma$ and $\Xi$. They first appear at density around twice nuclear saturation density. In these models the strength of the hyperonnucleon interaction is not calculated, but fitted to data, imposed as a variable parameter or taken the same - or similar to the N-N interaction. This uncertainty in the hyperon-nucleon potential influences directly the density dependence of the hyperon population. An example is shown in Figure $11[86,87]$ for two choices of the $U_{\Sigma}$ potential (attractive and repulsive), which significantly changes the threshold density for the $\Sigma$ hyperons. If the $\mathrm{U}_{\Sigma}$ potential was chosen to be negative (attractive), the lightest $\Sigma$ hyperon appears at $\sim 0.75 \mathrm{fm}^{-3}$ (left panel of Figure 11). Positive (repulsive) potential would move this threshold beyond densities relevant to cores of neutron stars. The $U_{\Xi}$ is attractive in both scenarios in Figure 11 causing the appearance of $\Xi$ hyperons at rather low densities, $\sim 0.4 \mathrm{fm}^{-3}$, even lower than the QMC prediction.

The composition of matter at densities expected in the cores of neutron stars has a decisive consequence for the maximum mass of a cold neutron star. The appearance of hyperons softens the EoS as was described in connection with Figure 10, which reduces the maximum neutron star mass. However, the fact that they appear only at higher densities in the QMC model means that the 
maximum mass predicted in this model is considerable higher that that in traditional models with hyperons. In Figure 12 we again show results for the N- (restricted nucleon only) and F- (full baryon octet) QMC700 models.. In most traditional models, this softening occurs at lower densities so that the predicted maximum mass falls below observation - the "hyperon puzzle" (see e.g. Figure 13 [89]).

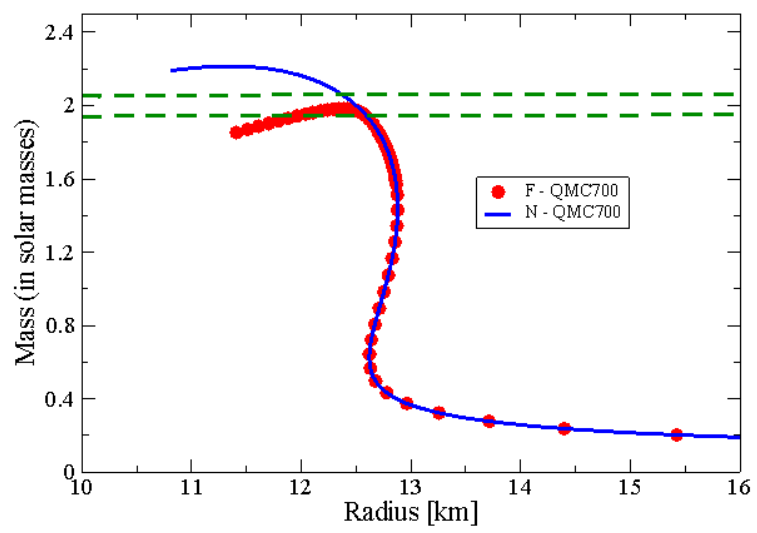

Figure 12. The gravitational masses of non-rotating neutron-star models (measured in solar masses) plotted against radius (in kilometers), calculated for F - QMC700 with hyperons (red dots) and N - QMC700 nucleonsonly (blue curve) [5]. The maximum mass of the star is $1.98 \mathrm{M} / \mathrm{M}$ with central density $0.82 \mathrm{fm}^{-3}$ and radius $12.38 \mathrm{~km}$. The horizontal green dashed lines depict the largest neutron star mass yet reported $[19,20]$.

Several remedies have been suggested to avoid this difficulty: introduction of three body hyperonhyperon forces [90], application of the Auxiliary Field Diffusion Monte Carlo (AFDMC) model to study N- $\Lambda$ potentials [91] or introducing an alternative theory of gravity [92]. The QMC model offers a simple solution of this puzzle in calculating the hyperon-nucleon consistently within its framework.

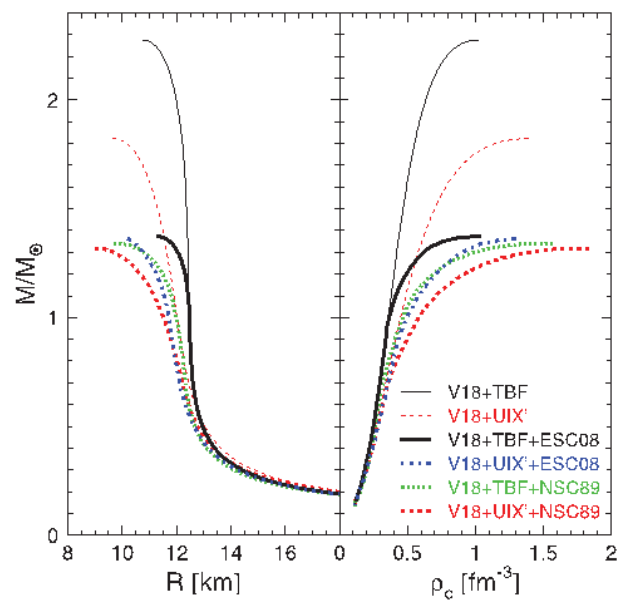

Figure 13. Neutron star mass - radius and mass - central density relations for different equations of state as calculated using the Bruckner-Hartree-Fock techniques with Nijmegen ESC08 hyperon-nucleon potentials. Thin curves V18+TBF and V18+UIX' equations of state are for nucleon only matter. Thicker lines are for the EoS of matter with nucleons, nuclear three-body forces and hyperons. The hyperon-nucleon potentials used are ESC08 and NSC89. Taken from [89]. 


\subsection{QMC and finite nuclei}

The first application of the QMC model to finite nuclei was performed by Guichon et a. [93] who calculated the charge density distribution in ${ }^{16} \mathrm{O}$ and ${ }^{40} \mathrm{Ca}$, and single-particle energies in ${ }^{40} \mathrm{Ca}$ finding good agreement with results of the RMF model of Walecka and Serot. Later, Guichon and Thomas [76] formulated the QMC model as a many-body effective Hamiltonian, which led naturally to the appearance of many-body forces. In the zero range limit of the model, its Hartree-Fock Hamiltonian was compared to that corresponding to the Skyrme effective force. By fixing the three parameters of the model to reproduce the binding and symmetry energy of nuclear matter, it was possible to map the main Skyrme parameters to corresponding QMC expressions and thus find very satisfactory interpretation of the Skyrme force. This particular Skyrme parameterizing was one of only five which satisfied all the constraints used by Dutra et al. [6] and appeared on the list of recommended Skyrme forces for use in nuclear structure modeling.

In a later paper [94], the similarity of the QMC effective interaction to the widely used SkM* force was utilized, in its non-relativistic form, to calculate the binding energies and charge distributions of doubly magic nuclei within the Hartree-Fock-Bogoliubov approach and the calculation extended to the properties of nuclei far from stability. The resulting predicted two neutron drip lines and shell quenching were ecouraging. However, in this work only spherically symmetrical nuclei could be considered and the three essential parameters of the model, the nucleon meson-coupling constants at zero density, were fixed to chosen values $\rho_{0}=0.16 \mathrm{fm}^{-3}$ and $\mathrm{E} / \mathrm{A}=-15.85 \mathrm{MeV}$, and symmetry energy of nuclear matter $\mathrm{J}=30 \mathrm{MeV}$.
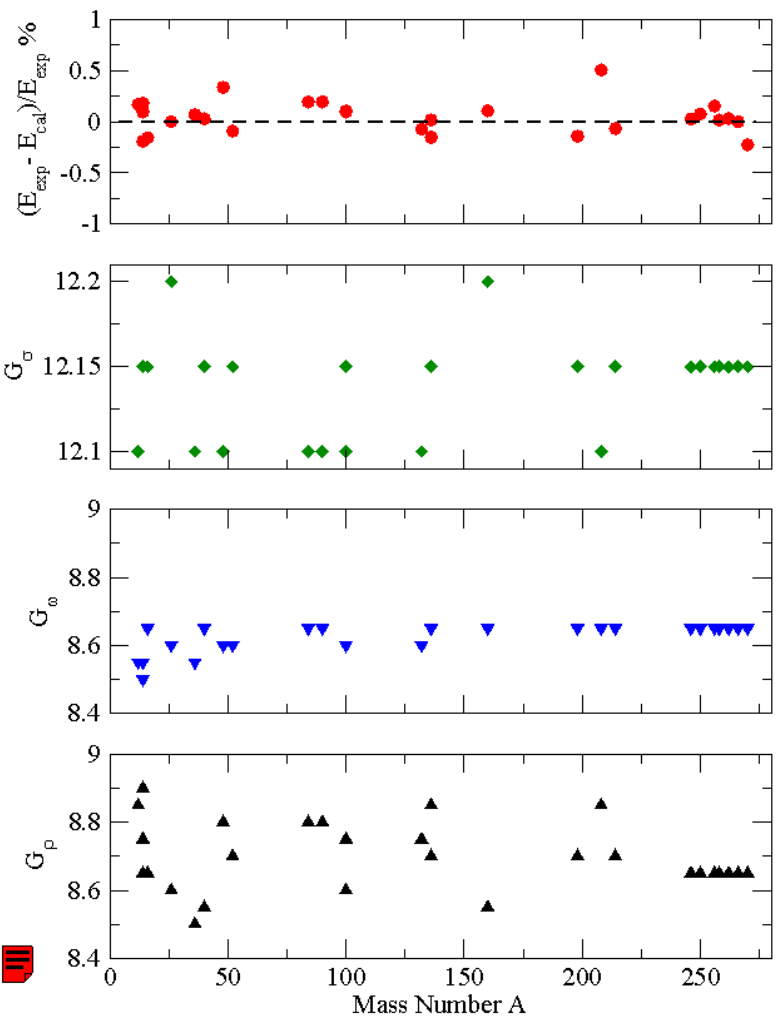

Figure 14. Preliminary (unpublished) results of the calculation of binding energies of even-even spherical and axially deformed nuclei in Hartree-Fock+BCS model with the QMC interaction. For more details see text. 
In the very recent development [95], the QMC non-relativistic Hamiltonian, implemented to an axial Hartree-Fock+BCS code to remove the limitation to spherical nuclei, was applied to investigate axially deformed nuclei. In this study, the allowed range of the three QMC parameters, Eq. 5, has been investigated in more detail. The parameters are correlated and it is possible to include the correlation in a search for their optimal values by exploring in a three-dimensional space consistent with the nuclear matter constrains, including their uncertainty. To set initial limits, the nuclear matter constraints were chosen to be:

$-17<\mathrm{E} / \mathrm{A}<-15 \mathrm{MeV}, 0.15<\rho_{0}<0.17 \mathrm{fm}^{-3}, 28<\mathrm{J}<34 \mathrm{MeV}, \mathrm{L}<25 \mathrm{MeV}$ and $250<\mathrm{K}_{\mathrm{o}}<350 \mathrm{MeV}$ ( $\mathrm{L}$ is the slope of the symmetry energy at saturation density). These limits constrained the QMC parameters uniquely to the ranges: $10.6<\mathrm{G}_{\sigma}<12.2 \mathrm{fm}^{2}, 7.4<\mathrm{G}_{\omega}<8.7 \mathrm{fm}^{2}$ and $7.2<\mathrm{G}_{\omega}<8.7 \mathrm{fm}^{2}$ and the investigation sought to further limit these ranges by fitting to the binding energy of even-even nuclei, ranging from ${ }^{14} \mathrm{C}$ to ${ }^{270} \mathrm{Ds}$.

A mesh technique was used in which the value of the binding energy is calculated for all combinations of the parameters in the parameter phase space, assuring that the true minimum has been reached, excluding any possibility of being trapped in a local minimum. As is shown in Figure 14 , fits to within of order $0.2 \%$ were achieved over the full range and the resulting parameter ranges are much reduced as shown in the lower panels of the figure with remarkable consistency from very light to superheavy nuclei. Preliminary data on axial deformation, root-mean-square radii, change density distribution and single particle energies are promising.

It is to be stressed that investigation of the optimum parameter set using the mesh technique is impracticable for effective models with many correlated parameters, such as the Skyrme forces.

\section{Summary and outlook.}

The main purpose of this review has been to critically assess the current status of knowledge of the properties of high-density matter above nuclear saturation density. Although there is some evidence for constraining matter models, particularly pure neutron matter, below that density, there is great uncertainty as to what happens at higher densities. Available astrophysical observational data, as well as data from terrestrial experiments, have insufficient accuracy and sensitivity to the microscopic composition of the matter, for the construction of a meaningful, well based, EoS.

We focused on the most relevant data input, neutron star masses, the simultaneous determination of masses and radii, and the cooling properties of neutron stars which provide the most direct evidence to better determine the EoS of high-density matter. Gravitational waves, although they may significant contribution are as yet unobserved.

The best terrestrial experiments, heavy-ion collisions, suffer from significant model dependence in their analysis and other terrestrial methods, for example parity violating electron scattering, antiprotonic atoms and determination of electric dipole strength function (see review e.g. [41]) have similar problems yielding limited relevance to EoS in stellar environments.

Review of the current main theoretical approaches to high-density matter found that they have many (correlated) parameters generally lacking clear physical meaning. The number of equivalent parameter combinations is practically infinite. Any time a new piece of experimental data appears, there is enough freedom in too many theoretical models so that parameter adjustment can obtain agreement with the data. Development of models for only local application having very limited or no predictive power in related phenomena is of little value.

We can anticipate progress on both experimental and theoretical fronts. On the astrophysics side, the new, now approved, NICER (The Neutron star Interior Composition ExploreR) [96] NASA mission promises to "explore the exotic states of matter inside these stars, where density and pressure are higher than in atomic nuclei, confronting theory with unique observational constraints. NICER will enable rotation-resolved spectroscopy of the thermal and non-thermal emissions of neutron stars in the soft (0.2-12 keV) X-ray band with unprecedented sensitivity, probing interior structure, the origins of dynamic phenomena, and the mechanisms that underlie the most powerful cosmic particle accelerators known". In addition, any signal from gravitational wave detectors would be most 
desirable [97]. On the terrestrial side, the FAIR and NICA accelerators can be expected to shed new light on the composition of matter created in heavy-ion collisions.

Finally, as an example of theoretical concepts alternative to the status quo, we gave space to a QMC model, which, although in existence for a rather long time, has not receive a lot of attention. And yet, it offers clear theoretical insight into medium effects on the structure of an individual hadron and the origin of many-body forces in matter over a usefully wide density range. Both effects are not well understood in current many-body models. The unique feature of the model is the limited number of parameters, well connected to physics and strongly constrained. Since there is no room to arbitrarily vary parameters in this model, any disagreement with new data indicates physics beyond the existing model.

The extreme conditions of high-density matter stretch both theory and experiment to their limits. Even partial solution of the challenges would constitute real progress in our understanding of Nature.

\section{References:}

1. F. Weber, Prog. Part. Nucl. Phys. 54, 193 (2005)

2. W. Weise, Prog. Part. Nucl. Phys, 67, 299(2012)

3. J. R. Stone and P.-G. Reinhard, Prog. Part. Nucl. Phys. 58, 341 (2007)

4. P. Ring, Prog. Part. Nucl. Phys. 37, 193 (1996)

5. J. R. Stone, P.A.M. Guichon, H.H. Matevosyan, A.W.Thomas, Nucl. Phys. A792, 341 (2007)

6. M. Dutra, O. Lourenco, J. S. Sa. Martins, A. Delfino, J. R. Stone, P. D. Stevenson, Phys. Rev. C 85, $035201(2012)$

7. M. Dutra, O. Lourenco, S. S. Avancini, B. V. Carlson, A. Delfino, D. P. Menzes, C. Provindencia, S. Typel, J. R. Stone, Phys. Rev. C 90, 055203 (2014)

8. Z. H. Li, U. Lombardo, H.-J. Schulze, W. Zuo, L. W. Chen, H. R. Ma, Phys. Rev. C 74, 047304 (2006)

9. T. Kruger, I. Tews, K. Hebeler, A. Schwenk, Phys. Rev. C 88, 025802 (2013)

10. H. Chen, M. Baldo, G. F. Burgio, H.-J. Schulze, Phys. Rev. D 86, 045006 (2012)

11. D. Logoteta, I. Bombaci, C. Providencia, I. Vidana, Phys. Rev. D 85, 023003 (2012)

12 A. Kurkela, P. Romatschke, A. Vuorinen, Phys. Rev. D 81, 105021 (2010)

13. S. Weissenborn, I. Sagert, G. Pagliara, M. Hempel, J. Schaffner-Bielich, ApJL 740, L14 (2011)

14. A. W. Steiner, J. M. Lattimer, E. F. Brown, ApJL 765, L5 (2013)

15. T. Fischer and M. Hempel, EPJ A 50, 46 (2014)

16. A. G. Lyne et al., Science 303, 20 (2004)

17. R. A. Hulse and J. H. Taylor, ApJL 195, L51 (1975)

18. P. C. C. Freire et al., MNRAS 412, 2763 (2011)

19. P. Demorest, T. Pennucci, M. S. E. Roberts. W. T. Hessels, Nature 467, 1081 (2010)

20. J. Antoniadis et al., Science 340, 448 (2013)

21. C. O. Heinke, Journal of Physics: Conference Series 432, 012001 (2013)

22. D. K. Galloway, M. P. Muno, J. M. Hartman, D. Psaltis, D. Chakrabarty, ApJS 179, 360 (2010)

23. F. Özel and D. Psaltis, Phys. Rev. D 80, 103003 (2009)

24. A. W. Steiner, J. M. Lattimer, E. F. Brown, ApJ 722, 33 (2010)

25. V. Suleimanov, J. Poutanen, M. Revnivtsev, K. Werner, ApJ 742, 122 (2011)

26. J. M. Lattimer and A. W. Steiner, arXiv:1305.3242 (2013)

27. S. Guillot, M. Servillat, N. A. Webb, R. E. Rutledge, ApJ 772, 7 (2013) .

28. J. M. Lattimer and A. W. Steiner, ApJ 784, 123 (2014)

29. J. Poutanen, J. Nattila, J. J. E. Kajava, O.-M. Latvala, D. Galloway, E. Kuulkers, V. Suleimanov, MNRAS 442, 3777 (2014)

30. C. O. Heinke et al., MNRAS 444, 443 (2014)

31. C. O. Heinke, J. E. Grindlay, D. A. Lloyd, P. D. Edmonds, arXiv:0301235 (2014)

32. W. C. G. Ho and C. O. Heinke, Nature 462, 71 (2009)

33. C. O. Heinke and W. C. G. Ho ApJL 719, L167 (2010)

34. D. Page, M. Prakash, J. M. Lattimer, A. W. Steiner, Phys. Rev. Lett. 106, 081101 (2011)

35. D. Blaschke, H. Grigorian, D. N. Voskresensky, F. Weber, Phys. Rev. C 85, 022802(R) (2012)

36. R. Negreiros, S. Schramm, F.Weber, Phys. Rev. D 85, 014019 (2012); 
R. Negreiros, S. Schramm, and F. Weber, Astron. Nachr. / AN 335, No. 6/7, 703 - 708 (2014);

37. D. G. Yakovlev, W. C. G. Ho, P. S. Shternin, C. O. Heinke, A. Y. Potekhin, MNRAS 411, 1977 (2011)

38. B. Posselt, G. G. Pavlov, V. Suleimanov, O. Kargaltsev, ApJ 779, 186 (2013)

39. B. Posselt, 2014cxo.prop.4399P

40. K. G. Elshamouty, C. O. Heinke, G. R. Sivakoff, W. C. G. Ho, P. S. Shternin, D. G. Yakovlev, D. J. Patnaude, L. David, ApJ 777, 22 (2013)

41. M. B. Tsang, J. R. Stone, et al., Phys. Rev. C 86, 015803 (2012)

42. P. Danielewicz, Nucl.Phys. A 661, 82 (1999)

43. P. Danielewicz, Nucl.Phys. A 673, 375 (2000)

44. P. Danielewicz, Roy Lacey, W. G. Lynch, Science 298,1592 (2002)

45. Bao-An Li, Lie-Wen Chen, Che Ming Ko, Phys. Rep. 464, 113 (2008)

46. C. Fuchs, Prog. Part. Nucl. Phys. 56, 1 (2009)

47. M. B. Tsang, Y. Zhang, P. Danielewicz et al., Phys. Rev. Lett. 102, 122701 (2009)

48. W. G. Lynch, M. B. Tsang, Y. Zhang, Prog. Part. Nucl. Phys. 62, 427 (2009)

49. T. Furuta and A. Ono, Phys. Rev. C 79, 014608 (2009)

50. R.H. Dalitz, A. Gal, Ann. Phys. 116, 167 (1978)

51. B. Povh, Prog. Part. Nucl. Phys. 18, 183 (1987)

52. Y. Yamamoto, T. Motoba, H. Himeno, K. Ikeda and S. Nagata, Prog. Theor. Phys. Suppl., 117, 361 (1994)

53. A. Gal, Prog. Theor. Phys. Suppl. 156, 1 (2004)

54. O. Hashimoto and H. Tamura, Prog. Part. Nucl. Phys. 57, 564 (2006)

55. P.A.M. Guichon, A.W.Thomas and K. Tsushima, Nucl.Phys. A 814, 66 (2008)

56. S. Bart, et al., Phys. Rev. Lett. 83, 5238 (1999)

57. H. Noumi, et al., Phys. Rev. Lett. 89, 072301 (2002);

H. Noumi, et al., Phys. Rev. Lett. 90, 049902 (2002), Erratum.

58. P. K. Saha, et al., Phys. Rev. C 70, 044613 (2004)

59. M. Troyer and U.-J. Wiese, Phys. Rev. Lett. 94, 170201 (2005)

60. V. G. J. Stoks, R. A. M. Klomp, C. P. F. Terheggen, and J. J. de Swart, Phys. Rev. C 49, 2950 (1994); R. B. Wiringa, V. G. J. Stoks, and R. Schiavilla, ibid. 51, 38 (1995); L. Engvik, M. Hjorth-Jensen, R. Machleidt, H. Muther, and A. Polls, Nucl. Phys. A 627, 85 (1997); R. Machleidt, Phys. Rev. C 63, 024001 (2001), and references therein.

61. B. ter Haar and R. Malfliet, Phys. Rep. 149, 207 (1987); E. N. E. van Dalen, C. Fuchs, A. Faessler, Nucl. Phys. A 741, 227 (2004); F. de Jong and H. Lenske, Phys. Rev. C 57, 3099 (1998).

62. J. Cugnon, P. Deneye, and A. Lejeune, Z. Phys. A 328, 409 (1987); W. Zuo, A. Lejeune, U. Lombardo, J. F. Mathiot, Eur. Phys. J. A 14, 469 (2002); I. Bombaci and U. Lombardo, Phys. Rev. C 44, 1892 (1991); B. D. Day and R. B. Wiringa, ibid. 32, 1057 (1985); M. Baldo, G. F. Burgio, and H.-J. Schulze, ibid. 61, 055801 (2000); I. Vidana, A. Polls, A. Ramos, L. Engvik, and M. Hjorth-Jensen, ibid. 62, 035801 (2000).

63. A. Akmal, V. R. Pandharipande, and D. G. Ravenhall, Phys. Rev. C 58, 1804 (1998).

64. A. Mukherjee and V. R. Pandharipande, Phys. Rev. C 75, 035802 (2007).60. A. Fabricioni and S. Fantoni, Phys. Lett. B 298, 263 (1993); C. Bisconti, F. A. de Saavedra, G. Co, and A. Fabrocini, Phys. Rev. C 73, 054304 (2006).

65. Y. Dewulf, W. H. Dickhoff, D. Van Neck, E. R. Stoddard, and M. Waroquier, Phys. Rev. Lett. 90, 152501 (2003).

66. T. Frick and H. Mu ther, Phys. Rev. C 68, 034310 (2003).

67. B. S. Pudliner, V. R. Pandharipande, J. Carlson, S. C. Pieper and R. B. Wiringa, Phys. Rev. C 56, 1720 (1997).

68. K. E. Schmidt and S. Fantoni, Phys. Letts. B 446, 99 (1999).

69. J. Carlson, J. Morales Jr., V. R. Pandharipande, and D. G. Ravenhall, Phys. Rev. C 68, 025802 (2003).

70. S. Gandolfi, F. Pederiva, S. Fantoni, and K. E. Schmidt, Phys.Rev. Lett. 99, 022507 (2007).

71. S. Gandolfi, A. Yu. Illarionov, S. Fantoni, F. Pederiva, and K. E. Schmidt, Phys. Rev. Lett. 101, 132501 (2008).

72. S. Gandolfi, A. Yu. Illarionov, K. E. Schmidt, F. Pederiva, and S. Fantoni, Phys. Rev. C 79, 054005 (2009). 
73. K. Hebeler and A. Schwenk, Phys. Rev. C 82, 014314 (2010).

74. K. Hebeler, J. M. Lattimer, C. J. Pethick, and A. Schwenk, Phys. Rev. Lett. 105, 161102 (2010).

75. P. A. M. Guichon, Phys. Letts. B 200, 235 (1988)

76. P. A. M. Guichon and A.W. Thomas, Phys. Rev. Lett. 93, 132502, (2004)

77. D. L. Whittenbury, J. D. Carroll, A. W. Thomas, K. Tsushima, J. R. Stone, Phys. Rev. C 89, 065801 (2014)

78. F. Bissey, F.-G. Cao, A. Kitson, B. G. Lasscock, D. B. Leinweber, A. I. Signal, A. G. Williams, J. M. Zanotti, Nucl. Phys. B (Proc. Suppl.) 141, 22 (2005) hep-lat/0501004.

79. F. Bissey et al., Phys. Rev. D 76, 114512 (2007)

80. T. Miyatsu, T. Katayama, K. Saito, Phys. Letts. B 709, 242 (2012)

81. T. Miyatsu, M.-K. Cheoun, K.Saito, Phys. Rev. C 88, 015802 (2013)

82. T. Miyatsu, M.-K. Cheoun, K.Saito, JPS Conf. Proc. 1, 013080 (2014)

83. J. Bartel, P. Quentin, M. Brack, C. Guet, and H.-B. Hakansson, Nucl. Phys. A 386, 79 (1982)

84. H.A. Bethe, M.B. Johnson, Nucl. Phys. A 230, 1 (1974).

85. J. R. Stone, N. J. Stone, S. A. Moszkowski, Phys. Rev. C 89, 044316 (2014)

86. J. Schaffner-Bielich, Nucl. Phys. A 835, 279 (2010)

87. J. Schaffner, I. N. Mishustin, Phys. Rev. C 53, 416 (1996)

89. H.-J.Schulze and T. Rijken, Phys. Rev. C 84, 035801 (2011)

90. I. Vidaña, D. Logoteta, C. Providência, A. Polls, I. Bombaci, EPL, 94, 11002 (2011)

91. D. Lonardoni, F. Pederiva, S Gandolfi, Journal of Physics: Conference Series, 529, 012012 (2014)

92. A. V. Astashenok, S. Capozziello, S. D. Odintsov, Phys. Rev. D 89, 103509 (2014)

93. P. A.M. Guichon,K. Saito, E. Rodionov, A. W. Thomas, Nucl. Phys. A 601, 349 (1996)

94. P. A. M. Guichon, H. H. Matevosyan, N. Sandulescu, A.W. Thomas, Nucl. Phys. A 772, 1 (2006)

95. J. R. Stone, P. A. M. Guichon, P.-G. Reinhard, A. W. Thomas (in preparation)

96. https://heasarc.gsfc.nasa.gov/docs/nicer/

97. A. Witze, Nature, 511, 2818 (2014) 